\title{
Current clinical trials update on HIV/AIDS: a systematic review
}

\author{
Harshul Batra, Shrikant Pawar \\ Georgia State University, United States
}

\begin{abstract}
Several clinical trials using different interventions are being sponsored to combat human immunodeficiency virus (HIV) at different stages. For evidence-based knowledge studies in medicine, clinical trials are of utmost importance. A legislative requirement to register the clinical trials at the ClinicalTrials.gov provides the information necessary for intensive evaluation, which was previously not possible. The purpose of this study was to provide an intensive portfolio of HIV clinical trials and perform a retrospective ClinicalTrials.gov data review. All active, open, and recruiting clinical trials registered at ClinicalTrials.gov up to May 2018 were included. Information related to trial characteristics, intervention type, primary outcome, and patient enrollment timeline was extracted. Information related to 10,182 registered HIV trials was download from ClinicalTrials.gov. Phase 2 clinical trials were the major ones comprising nearly $1.6 \%$ of total clinical trials with the industry being the major sponsor followed by NIH. Other characteristics analyzed included the number of trial centers, primary outcome, treatment setting, and time relation with phases. Common intervention were classified into adjuvant, non-adjuvant, and radiotherapy. The clinical trials data analysis provides a comprehensive description of HIV trials. The information provided may be useful to re-tailor the intervention techniques and to overcome the discrepancy in data management for clinical trials which would improve clinical trial design, and reduce failures and cost of trials.
\end{abstract}

HIV AIDS Rev 2019; 18, 2: 79-84 DOI: https://doi.org/10.5114/hivar.2019.86371

Key words: clinical trials, HIV, AIDS, ClinicalTrials.gov, data interpretation.

\section{Introduction}

Zoonotic infections with simian immunodeficiency viruses from African primates led to the human immunodeficiency virus (HIV) epidemic. The first group to be infected with HIV were bushmeat hunters. Apes and sooty mangabey monkeys were the source of transmission for HIV-1 and HIV-2 respectively [1]. There are several factors that increase risk of sexual transmission of HIV-1. The major one is the number of copies per ml of plasma HIV-1 RNA (viral load). With every $1 \log 10$ increase there is 2.4 times increased risk of sexual transmission [2]. Other factors

include pregnancy [3], sexually transmitted infections (herpes simplex type 2 infection [4], genital ulcers [5], and bacterial vaginosis [6]), and receptive anal intercourse [7]. Multiple sexual partners [8] and concurrent partnerships [9] are among the behavioral factors associated with increased HIV-1 sexual transmission, whereas male circumcision is associated with a reduced risk of sexual transmission of HIV [10].

HIV continues to be a major global public health issue, with nearly 78 million people infected since the start of the epidemic. Moreover, an estimated 35 million people have died of AIDS-related illnesses. 1,122,900 adults and

Article history:

Received: 09.10.2018

Received in revised form: 26.11 .2018

Accepted: 11.02.2019

Available online: 04.06.2019
International Journal of HIV-Related Problems

HIV \& AIDS

R e vi e w 
adolescents were living with HIV at the end of 2015, and 162,500 (15\%) of those had not received a diagnosis. Among people aged 13-24 who were living with HIV, around 44\% did not know about their infection. In 2016, 18,160 people received an AIDS diagnosis and since the epidemic began in the early 1980s, 1,232,346 people have received an AIDS diagnosis. Gay and bisexual men have accounted for $67 \%$ $(26,570)$ of all HIV diagnoses and $83 \%$ of diagnoses among males. Black/African American and bisexual men accounted for the largest number of HIV diagnoses $(10,223)$, followed by Hispanic/Latino $(7,425)$ and white $(7,390)$ gay and bisexual men. From 2011 to 2015, diagnoses decreased by $10 \%$ amongst white gay and bisexual men. Among Hispanic/Latino gay and bisexual men, diagnoses increased by $14 \%$. Heterosexual contact (injecting drugs) accounted for $24 \%(9,578)$ of HIV diagnoses. Women accounted for $19 \%(7,529)$ of HIV diagnoses [11, 12].

The Centers for Disease Control and Prevention (CDC) uses data from the National HIV Surveillance System and the Medical Monitoring Project to estimate the percentages of persons living with HIV infection and treated with antiretroviral drugs (ART). According to 2011 data, an estimated $40 \%$ of HIV diagnosed patients were engaged in HIV medical care, $37 \%$ were prescribed ART, and 30\% achieved viral suppression. The effect of ART therapy and viral suspension varied among different age groups with persons aged $\geq 65$ years showing the highest viral suspension (37\%) compared to $18-24$ years (13\%), 25-34 years (23\%), and 35-44 years (27\%) age group patients [13]. Improved health, prolonged lives, and prevention of transmission are benefits associated with ART. Future research work is focused on developing therapeutic strategies to induce sustained ART-free remission by employing an approach known as analytical treatment interruption [14].
Funding is another issue associated with HIV and AIDS. In recent years, a plateauing of global funding towards HIV and AIDS has been seen, with US\$ 19 billion invested among low- and middle-income countries [12]. From 2006 to 2016 domestic investment (increasing on average of $11 \%$ ) by several countries has pushed to overcome the insufficient international funding issue. Future commitment suggestions to overcome the HIV and AIDS epidemic have been estimated by the United Nations General Assembly at US\$ 23.9 billion in 2020 and with US\$ 23.9 billion required in 2030 [12].

We provide a global overview of the clinical trial statistics in HIV/AIDS infection. We briefly describe different characteristics for trial design and type of interventional studies used for HIV/AIDS infection. Finally, we provide a time-line of patient enrollment and registered patents for lung cancer and discuss the advantages, challenges, and perspectives for the improvement of clinical trial design for HIV/AIDS studies.

\section{Material and methods}

\section{Data source}

On March 1, 2018, a data set of 10,182 clinical studies related to HIV/AIDS was downloaded from ClinicalTrials.gov. The dataset was analyzed using various parameters in Excel. Information regarding terminologies can be obtained from the Clinical Trials Transformation Initiative website [15].

\section{Study design and parameters}

The data were restricted to active recruiting studies. In the advanced search option the following parameters were selected: "open/recruiting/active studies for recruitment sta-
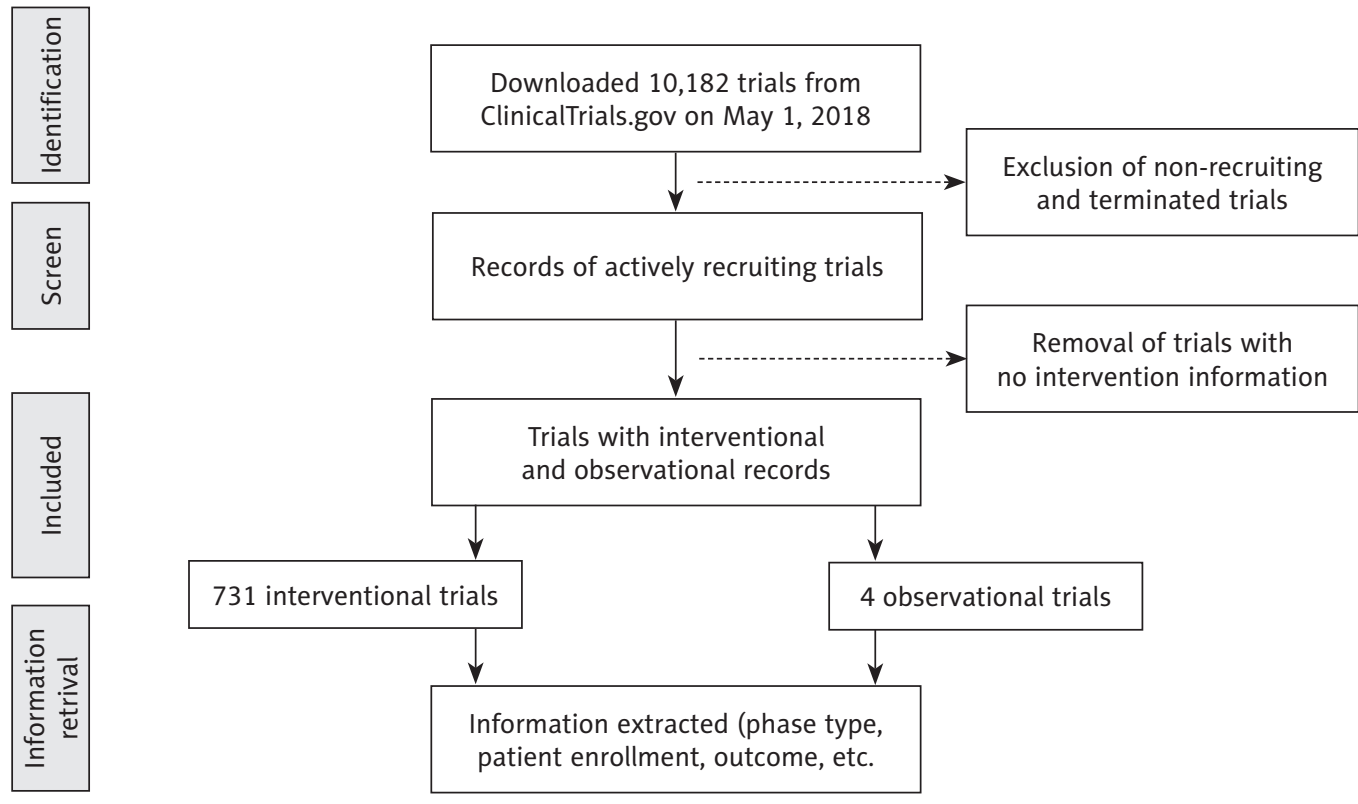

Figure 1. Flowchart of study selection 
tus", "all groups" for study, and age groups. The data extracted comprised both interventional and observational studies. We wanted to keep this review current, so we excluded all completed/terminated/not recruiting clinical trials from our search. Further clinical trials with no intervention information were excluded. The information contains all active clinical trials on HIV/AIDS until May 2018 in the ClinicalTrials. gov database. Each study was manually reviewed by the authors (title, interventions, outcome measures, recruiting status, MeSH terms, and the full ClinicalTrials.gov record if necessary) to ascertain relevance to HIV/AIDS study. Figure 1 depicts the workflow selected for final data extraction for analysis.

\section{Data collection and analysis}

The following information was extracted from the website: (1) clinical trial phase (early phase $1,1 / 2,2,2 / 3,3,4$ ) (2) recruiting status, (3) location of clinical center, (4) study design, (5) type of study (interventional, observational or others), (6) number of trial centers, (7) primary sponsor, (8) primary outcome, (9) treatment setting, (10) treatment classes, (11) time relation with phases. Along with that we also compiled information about patents related to HIV/ AIDS using HIV/AIDS treatment, HIV/AIDS therapeutic, and HIV/AIDS diagnostic. The information extracted was: (1) number of patents published, (2) patent office location, (3) primary applicant name, and (4) biologicals.

\section{Results}

\section{Trial characteristics and design}

Our parameters identified 10,182 trials involving HIV/ AIDS. 1161 had no treatment information or missing information regarding a location of clinical trials and were excluded from the total clinical trials identified. Overall, 382 (3.7\%) were actively recruiting, 110 (1.08\%) were not yet recruiting, and $19(0.1 \%)$ were enrolling by invitation only. For 323 trials (3.1\%) no information regarding the recruiting status was available, but we included them in our data analysis studies as these clinical trials showed up in our advanced search option when we selected for open and active studies. The characteristic numbers of these phases were as follows: Phase 1 trials $(159,1.5 \%)$, Phase $1 / 2(56,0.5 \%)$, Phase $2(168,1.6 \%)$, Phase $2 / 3(35,0.3 \%)$, Phase 3 (170, $1.6 \%)$, and Phase $4(149,1.4 \%)$. The major sponsor for clinical trials was the $\mathrm{NIH}$, accounting for $2.2 \%$ of trials. More than half of total clinical trials 794 (7.7\%) were conducted in the US, whereas those outside the US comprised the remaining $902(8.2 \%)$ clinical trials. A major portion of trials were interventional type, 731 (7.1\%), indicative of proper treatment/drug provided to one or more group to test for its effects. Moreover, as clinical trials are committing in cost, workload, and recruitment, more than half of the clinical trials were conducted at multiple locations (Table 1). A major proportion of the phase 2 (phase 1
Table 1. All open clinical trials characteristics. Data generated from ClinicalTrials.gov

\begin{tabular}{|c|c|}
\hline Factor & $\begin{array}{c}\text { No. of trials (\%) } \\
\text { Total: } 10,182\end{array}$ \\
\hline \multicolumn{2}{|l|}{ Type of clinical trials } \\
\hline Phase 1 & $159(1.5)$ \\
\hline Phase $1 / 2$ & $56(0.5)$ \\
\hline Phase 2 & $168(1.6)$ \\
\hline Phase $2 / 3$ & $35(0.3)$ \\
\hline Phase 3 & $170(1.6)$ \\
\hline Phase 4 & $149(1.4)$ \\
\hline Unspecified & $1161(11)$ \\
\hline \multicolumn{2}{|l|}{ Primary sponsor } \\
\hline Industry & $232(2.2)$ \\
\hline $\mathrm{NIH}$ & $188(1.8)$ \\
\hline Thai Red Cross AIDS Research Centre & $1(0.009)$ \\
\hline $\begin{array}{l}\text { Royal Thai Army Clinical Research } \\
\text { Center }\end{array}$ & $1(0.009)$ \\
\hline AFRIMS & $1(0.009)$ \\
\hline Bang Lamung District Hospital & $1(0.009)$ \\
\hline Emory University & $1(0.009)$ \\
\hline Botswana Ministry of Health clinics & $1(0.009)$ \\
\hline Brooke Army Medical Center & $1(0.009)$ \\
\hline VA Portland Health Care System & $1(0.009)$ \\
\hline Dallas VA Medical Center & $1(0.009)$ \\
\hline Infectious Diseases Institute & $1(0.009)$ \\
\hline Naval Medical Center San Diego & $1(0.009)$ \\
\hline Chulalongkorn University Hospital & $2(0.01)$ \\
\hline Eastern Virginia Medical School & $1(0.009)$ \\
\hline Others & $344(3.3)$ \\
\hline
\end{tabular}

Recruiting status

\begin{tabular}{l|c}
\hline Actively recruiting & $382(3.7)$ \\
\hline Not yet recruiting & $110(1.08)$ \\
\hline Active, not recruiting & $224(2.1)$ \\
\hline Enrolling by invitation & $19(0.1)$ \\
\hline Unknown & $323(3.1)$
\end{tabular}

Study locations

\begin{tabular}{l|c}
\hline Single & $1114(10.9)$ \\
\hline Multiple & $582(5.71)$ \\
\hline Unspecified & $203(1.99)$ \\
\hline
\end{tabular}

\begin{tabular}{l|l}
\hline Location of trial centers & \\
\hline Within US & $794(7.7)$ \\
\hline Outside US & $902(8.8)$
\end{tabular}

\begin{tabular}{l|c}
\hline \multicolumn{1}{l}{ Type of study } & \multicolumn{1}{l}{$902(8.8)$} \\
\hline Interventional & $731(7.1)$ \\
\hline Observational & $4(0.03)$ \\
\hline Others & $0(0)$ \\
\hline
\end{tabular}


Table 2. Characteristics of phase II and III clinical trials

\begin{tabular}{|c|c|c|c|c|}
\hline Study design & $\begin{array}{c}\text { No. of phase I } \\
\text { trials (\%) }\end{array}$ & $\begin{array}{c}\text { No. of phase II } \\
\text { trials (\%) }\end{array}$ & $\begin{array}{c}\text { No. of phase III } \\
\text { trials (\%) }\end{array}$ & $\begin{array}{c}\text { No. of phase IV } \\
\text { trials (\%) }\end{array}$ \\
\hline \multicolumn{5}{|l|}{ Randomization } \\
\hline Randomized & $162(1.5)$ & $127(1.2)$ & $130(1.2)$ & $101(0.99)$ \\
\hline Non-randomized trials & $41(0.4)$ & $21(0.2)$ & $11(0.1)$ & $14(0.13)$ \\
\hline Open label & $24(0.2)$ & $22(0.2)$ & $20(0.1)$ & $8(0.07)$ \\
\hline Single-blinded & $100(0.9)$ & $75(0.7)$ & $47(0.4)$ & $49(0.4)$ \\
\hline Double-blinded & $19(0.1)$ & $19(0.1)$ & $26(0.2)$ & $4(0.03)$ \\
\hline \multicolumn{5}{|l|}{ Number of treatment arms } \\
\hline Single arm & $1(0.009)$ & $0(0)$ & $0(0)$ & $0(0)$ \\
\hline Two arms & $0(0)$ & $0(0)$ & $0(0)$ & $0(0)$ \\
\hline Three or more & $0(0)$ & $1(0.009)$ & $0(0)$ & $0(0)$ \\
\hline \multicolumn{5}{|l|}{ Type of control arm } \\
\hline Placebo & $55(0.5)$ & $51(0.5)$ & $34(0.33)$ & $13(0.1)$ \\
\hline Standard care or active control & $0(0)$ & $0(0)$ & $0(0)$ & $1(0.009)$ \\
\hline \multicolumn{5}{|l|}{ Primary outcome } \\
\hline Overall survival & $10(0.09)$ & $20(0.1)$ & $9(0.08)$ & $0(0)$ \\
\hline Progression-free survival & $3(0.02)$ & $4(0.03)$ & $1(0.009)$ & $0(0)$ \\
\hline Quality of life & $3(0.02)$ & $11(0.1)$ & $24(0.2)$ & $13(0.1)$ \\
\hline Disease-free survival & $0(0)$ & $6(0.05)$ & $2(0.01)$ & $0(0)$ \\
\hline
\end{tabular}

A
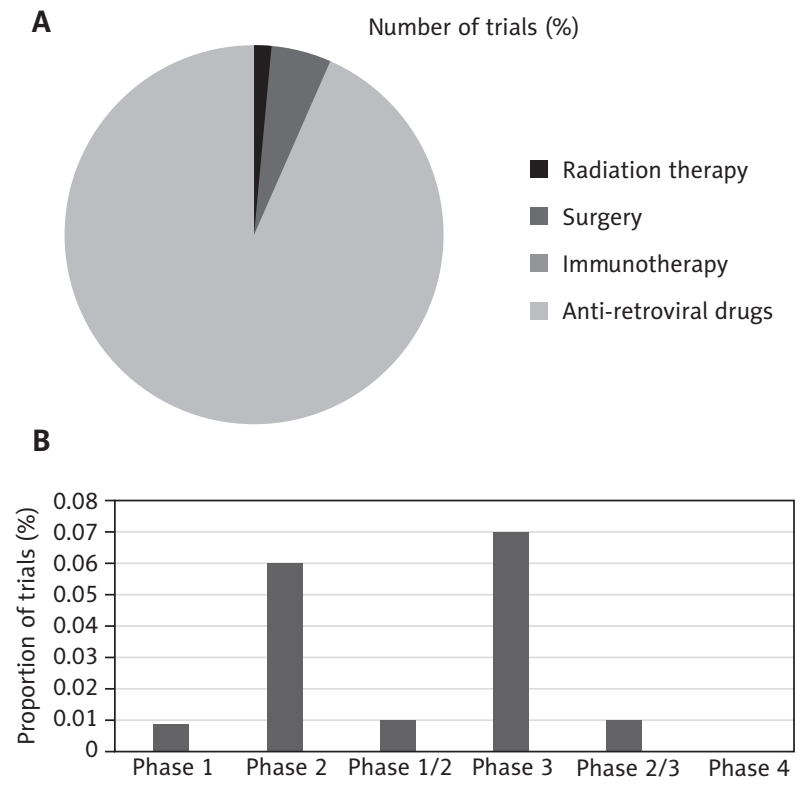

Figure 2. Types of treatment for HIV in clinical trials phase I-IV. A) Types therapy for treating HIV. B) Biomarker analysis studies in clinical trials

+ phase $1 / 2)$ trials were randomized (1.5\%) or single-blinded $(0.9 \%)$. For phase 1 and phase $2(0.009 \%)$ the standard care or active control was the major arm (Table 2). Overall survival was the major primary outcome in all phases
A

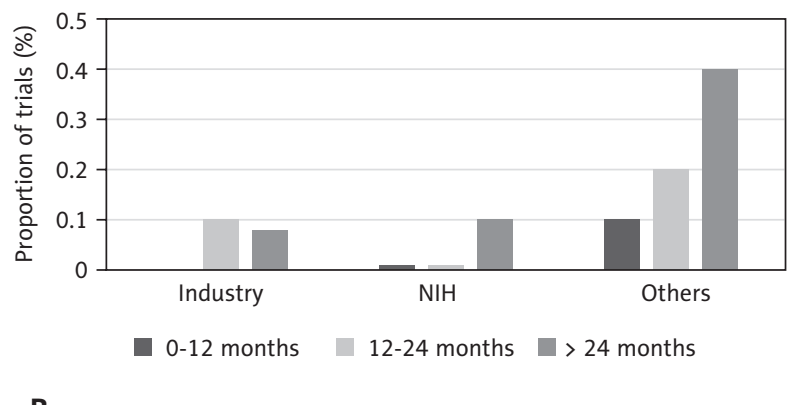

B

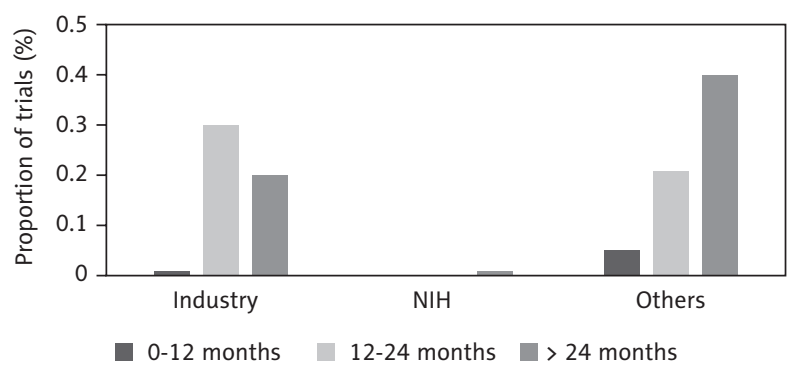

Figure 3. Clinical trials time line for patient enrollment. A) Phase II and B) phase III

of trials (phase 1, 0.09\%; phase 2, 0.1\%; phase 3, 0.08\%) followed by progression-free survival (phase 1, 0.02\%; phase $2,0.03 \%$; phase $3,0.009 \%$ ), and quality of life (phase 1 , $0.02 \%$; phase $2,0.05 \%$; and phase $3,0.01 \%$ ) (Table 2 ). 


\section{Intervention type and treatment settings}

HIV/AIDS treatment involves several types of therapeutic methods as seen in Figure 2. Adjuvant therapy was the most used intervention for nearly $0.06 \%$ of clinical trials. This was followed by non-adjuvant therapy $(0.03 \%)$ followed by second line and radiotherapy comprising $0.02 \%$ and $0.01 \%$ clinical trials respectively (Table 3 and Table 4, Figure 2A).

\section{Biomarker analysis}

Early detection of HIV/AIDS plays a key role in successful treatment. Recently, several research studies have focused on identifying and detecting specific biomarkers in HIV/AIDS. We also analyzed data from all phases and found out that for 36 clinical trials as a study objective a biomarker was included. In clinical trials biomarker information was provided for (Figure 2B) Phase $1(1,0.009 \%)$, Phase 2 (7, 0.06\%), Phase $1 / 2(2,0.01 \%)$, Phase 3 (8,0.07\%), and Phase $2 / 3(2,0.01 \%)$

\section{Patient enrollment timeline}

Patient enrollment in Phase 2 and Phase 3 clinical trials were also taken into consideration. The success of results generation of a clinical trial depends on statistical analysis of the number and time of patient enrollments. Industry-sponsored Phase 2 clinical trial were open for more than 2 years $(0.08 \%)$ and were of smaller proportion compared to NIH sponsored clinical trials. Phase 3 clinical trials had a similar trend with the NIH-sponsored trial taking the lead along with nearly $0.1 \%$ compared to industry-sponsored clinical trials. Again, patient enrollment was not provided for a large number of NIH sponsored Phase 3 clinical trials (Figure 3).

\section{Discussion}

Our contemporary survey provides a landscape of registered HIV/AIDS clinical trials including interventional and observational studies. Various characteristics of trials such as design, location, type of intervention, patient enrolment, and sponsors are discussed. Several noteworthy observations emerge from this review of clinical trials of HIV/AIDS. The survey suggested the majority of trials on the ClinicalTrials.gov website were phase 2 . As setting up clinical trials requires intensive utilization of resources, money, and patient enrolment, HIV/AIDS trials were majority multi-centered and were sponsored by industry, NIH, and universities. In our analysis, several data points were unspecified as the trial lacked the information for that specific column. NIH phase 2 clinical trials were open for a longer time compared to industry-sponsored trials.

One of the major problems with all these clinical trials was the absence of biomarkers in phase 2 trials, and a similar pattern is followed at phase 3 lung cancer trials. For
Table 3. Chemotherapy treatment settings and biomarkers for clinical trials

\begin{tabular}{l|c}
\hline Treatment setting & No. of trials (\%) \\
\hline Adjuvant therapy & $7(0.06)$ \\
\hline Neoadjuvant therapy & $4(0.03)$ \\
\hline Adjuvant/neoadjuvant therapy & $0(0)$ \\
\hline Radiotherapy & $2(0.01)$ \\
\hline Advanced-stage disease & $2(0.01)$ \\
\hline First line & $0(0)$ \\
\hline First or second line & $3(0.02)$ \\
\hline Second line & $8(0.07)$ \\
\hline Maintenance & $36(0.3)$ \\
\hline Biomarker(s) specified & $1(0.009)$ \\
\hline Yes & $7(0.06)$ \\
\hline Phase of trial & $2(0.01)$ \\
\hline Phase 1 & $8(0.07)$ \\
\hline Phase 2 & $2(0.01)$ \\
\hline Phase 1/2 & $0(0)$ \\
\hline Phase 3 & \\
\hline Phase 2/3 & \\
\hline Phase 4 & \\
\hline
\end{tabular}

Table 4. Types of treatment for HIV in clinical trials

\begin{tabular}{l|c}
\hline Treatment & Number of trials (\%) \\
\hline Radiation therapy & $9(0.08)$ \\
\hline Surgery & $31(0.3)$ \\
\hline Immunotherapy & $3(0.02)$ \\
\hline Anti-retroviral drugs & $590(5.7)$ \\
\hline
\end{tabular}

only $0.3 \%$ of total clinical trials biomarker analysis information was available. For future success, early-stage clinical trials aiming at the better understanding of pathways and molecular level studies need to be implemented before moving forward with large clinical trials. Moreover, biomarker analysis collection needs to be an active step in future development. Enrolling more patients for early-stage HIV/AIDS would be a good way to overcome this limitation. The biomarker selection for molecularly targeted therapy and development of novel therapy/biomarker using studies performed on tissue samples could dramatically improve the cure rates in HIV/AIDS. Other important factors to consider when designing clinical trials are mainly related to the complex and difficult ethical challenges. These mainly include scientific validity for clinical trials, fairness in the selection of study sites and participants, consideration of risk/benefit ratio, independent ethical and scientific review, and informed consent $[16,17]$. 


\section{Conclusions}

Based on the data collected from ClinicalTrials.gov, our analysis reveals that the majority of clinical trials were phase 2, with NIH trials lasting for more than 2 years of enrollment and radiation, surgery and chemotherapy being the major interventions. Most of them were randomized trials with the primary outcome focused on patient survival. Moreover, if successful interventions related to HIV are found in clinical trials then efforts should be made to direct funding towards patients from low economy countries. Our comprehensive analysis provides useful information regarding HIV/AIDS which may be helpful to industry and investigators for future decisions.

\section{Conflict of interest}

The authors declare no potential conflicts of interest with respect to the research, authorship, and/or publication of this article.

\section{References}

1. Sharp PM, Hahn BH. Origins of HIV and the AIDS pandemic. Cold Spring Harb Perspect Med 2011; 1: 006841.

2. Quinn TC, Wawer MJ, Sewankambo N, et al., and the Rakai Project Study Group. Viral load and heterosexual transmission of human immunodeficiency virus type 1. N Engl J Med 2000; 342: 921-929.

3. Mugo NR, Heff ron R, Donnell D, et al., and the Partners in Prevention HSV/HIV Transmission Study Team. Increased risk of HIV-1 transmission in pregnancy: a prospective study among African HIV-1serodiscordant couples. AIDS 2011; 25: 1887-1895.

4. Glynn JR, Biraro S, Weiss HA. Herpes simplex virus type 2: a key role in HIV incidence. AIDS 2009; 23: 1595-1598.

5. Røttingen JA, Cameron DW, Garnett GP. A systematic review of the epidemiologic interactions between classic sexually transmitted diseases and HIV: how much really is known? Sex Transm Dis 2001; 28: 579-597.

6. Atashili J, Poole C, Ndumbe PM, et al. Bacterial vaginosis and HIV acquisition: a meta-analysis of published studies. AIDS 2008; 22: 1493-1501.

7. Baggaley RF, White RG, Boily MC. HIV transmission risk through anal intercourse: systematic review, meta-analysis and implications for HIV prevention. Int J Epidemiol 2010; 39: 1048-1063.

8. Tanser F, Bärnighausen T, Hund L, et al. Effect of concurrent sexual partnerships on rate of new HIV infections in a high-prevalence, rural South African population: a cohort study. Lancet 2011; 378: 247-255.

9. Epstein H, Morris M. Concurrent partnerships and HIV: an inconvenient truth. J Int AIDS Soc 2011; 14: 13.

10. Weiss HA, Quigley MA, Hayes RJ. Male circumcision and risk of HIV infection in sub-Saharan Africa: a systematic review and meta-analysis. AIDS 2000; 14: 2361-2370.

11. Centers for Disease Control. HIV in the United States: At a glance. 2018. Available at: https://www.cdc.gov/hiv/statistics/overview/ataglance.html.

12. Joint United Nations Programme on HIV/AIDS (UNAIDS). Global HIV statistics. Fact sheet July, 2017.

13. Bradley B, Hall H, Wolitski R, et al. Vital signs: HIV diagnosis, care, and treatment among persons living with HIV - United States, 2011. MMWR Morb Mortal Wkly Rep 2014; 63: 1113-1117.

14. Clarridge K, Blazkova J, Einkauf K, et al. Effect of analytical treatment interruption and reinitiation of antiretroviral therapy on HIV reservoirs and immunologic parameters in infected individuals. PLoS Pathog 2018; 14: e1006792.

15. ClinicalTrials.gov. Protocol Data Element Definitions, 2015. Available at: https://prsinfo.clinicaltrials.gov/definitions.html.

16. Lo B, Grady C. Ethical considerations in HIV cure research: points to consider. Curr Opin HIV AIDS 2013; 8: 243-249.

17. Eyal N. The benefit/risk ratio challenge in clinical research, and the case of HIV cure: an introduction. J Med Eethics 2017; 43: 65-66. 